\title{
REDISEÑO CURRICULAR EN LA UNIVERSIDAD DE CONCEPCIÓN: LA EXPERIENCIA DE LAS CARRERAS DE FORMACIÓN INICIAL DOCENTE ${ }^{1}$
}

\author{
Cecilia Cisterna ${ }^{2}$ \\ Valentina Soto ${ }^{3}$ \\ Constanza Rojas ${ }^{4}$
}

\begin{abstract}
RESUMEN
El objetivo de este trabajo fue describir y analizar la experiencia de rediseño curricular de las 19 carreras de pedagogía en la Universidad de Concepción. Este proceso se realizó gracias a un Convenio de Desempeño en el ámbito de formación de profesores, suscrito entre la universidad y el Ministerio de Educación de Chile. En el primer apartado, se detalla el contexto en el cual se realizó el rediseño: las transformaciones en la formación universitaria, la introducción del concepto de competencias y resultados de aprendizaje, así como los desafíos de la formación inicial docente. En el segundo apartado, se presenta la experiencia de rediseño curricular a partir de antecedentes que permiten comprender su origen y desarrollo, y luego se describen las fases del rediseño, relevando tanto el proceso como los resultados. Finalmente, se analizan las principales innovaciones en tres aspectos (legales o administrativos, institucionales y de docencia) relacionándolas con la experiencia del rediseño curricular.
\end{abstract}

Palabras clave: competencias, formación de profesores, plan de estudios, rediseño curricular, resultados de aprendizaje.

\section{CURRICULUM REDESIGN AT UNIVERSITY OF CONCEPCIÓN: THE EXPERIENCE OF INITIAL TEACHER TRAINING PROGRAMS}

\section{ABSTRACI}

The main aim of this article is to describe and analyze the curriculum redesign experience of 19 teacher education programs of the University of Concepción. This process was developed in the context of a performance agreement in the area of teacher training, subscribed between the Ministry of Education and the university. In the first section, information is provided about the context in which the curriculum redesign occurred, in regards to changes in university education, the incorporation of the concept of competencies and learning outcomes, and the challenges of initial teacher training. In the second part, the origin and development of the curriculum redesign experience are presented, followed by a description of the multiple stages, highlighting the process as well as the results. The paper concludes with an analysis of the legal or administrative, institutional and academic aspects of the primary innovations resulting from the curriculum design experience.

Keywords: competency, curriculum redesign, learning outcomes, study plans, teacher training.

1 Este trabajo se llevó a cabo en el marco del Convenio de Desempeño en Formación de Profesores "Profesores UdeC: protagonistas del cambio en la sociedad del conocimiento", UCO1203, Universidad de Concepción/ Ministerio de Educación.

2 Facultad de Humanidades y Arte, Universidad de Concepción, Concepción, Chile. Contacto: cecisterna@udec.cl

3 Dirección de Docencia, Universidad de Concepción, Concepción, Chile. Contacto: valentinasoto@udec.cl

4 Dirección de Docencia, Universidad de Concepción, Concepción, Chile. Contacto: constanzarojas@udec.cl 


\section{El Proceso de Bolonia y la formación por competencias}

El sistema de educación superior se encuentra en un período de redefinición del proceso de enseñanza, con el fin de promover experiencias significativas de aprendizaje para los estudiantes. En este contexto, se firmó en el año 2000 la Declaración de Bolonia que propuso un marco común de enseñanza y aprendizaje en el sistema de educación superior europeo. Sus principales iniciativas se orientaron a la armonización de la arquitectura curricular, la implementación de créditos transferibles y el reconocimiento de las cualificaciones académicas. Producto de esta declaración, se instituyó el Tuning Educational Structures in Europe $e^{5}$ (conocido como proyecto Tuning), un proyecto que entrega lineamientos para implementar los principios de Bolonia.

El proyecto Tuning propone el concepto de competencias y resultados de aprendizaje como elementos clave para el diseño de los planes de estudio, las metodologías de enseñanza y los procesos evaluativos.

Los resultados de aprendizaje son formulaciones de lo que el estudiante debe conocer, comprender o ser capaz de demostrar tras la finalización del proceso de aprendizaje, ... las competencias se definen como una combinación dinámica de conocimientos, comprensión, habilidades y capacidades (Universidad de Deusto, 2006, p. 8).

En efecto, este enfoque responde a una concepción del perfil profesional que favorece el desempeño autónomo, el pensamiento crítico, las habilidades de comunicación, el dominio de otros idiomas, la ética y el autoaprendizaje (Hambur, Rowe \& Lucgrew, 2002).

El Sistema Europeo de Transferencia de Créditos, otra de las principales propuestas del proyecto Tuning, establece una relación directa entre las competencias, los resultados de aprendizaje y la carga real de trabajo del estudiante requerida

5 Conocido también como "Afinar las estructuras educativas en Europa". 
para completar cada una de las actividades planificadas (Dochy \& Nickmans, 2005). En Chile, se adoptó el Sistema de Créditos Académicos Transferibles (SCT), a partir de una serie de iniciativas que surgieron del Programa Mecesup y del Consejo de Rectores de las Universidades Chilenas (CRUCH), que promovieron su implementación en los procesos de rediseño curricular con el objetivo de mejorar la legibilidad de los programas de estudio, conocer la demanda real del trabajo académico desarrollado por los estudiantes en las distintas asignaturas del plan de estudio y equilibrar la carga académica, además de promover la movilidad estudiantil en Chile y en el extranjero. A partir de este nuevo enfoque, el énfasis se centró en el logro de aprendizajes de los estudiantes en una carga académica comparable (CRUCH, 2013).

\subsection{Desafíos en la formación de profesores}

La formación de los futuros profesores se encuentra presente en el debate nacional e internacional en función de la centralidad del rol docente en el mejoramiento de la educación de niños y jóvenes. Los informes de la Comisión sobre Formación Inicial Docente (Navarro, 2005) y de la Organisation for Economic Co-operation and Development, OECD (2011) coinciden en la identificación de algunas debilidades de la formación de profesores en Chile, entre las cuales se destacan: la carencia de una orientación hacia logros medibles, un escaso desarrollo de la innovación curricular, falta de investigación de los formadores de profesores acerca de su propio quehacer y escasa articulación entre la formación pedagógica y la formación en la especialidad.

Estos nudos críticos son similares a los que enfrenta la formación inicial docente en Latinoamérica. De acuerdo con Vaillant (2013), los problemas más relevantes se asocian con la falta de preparación de los candidatos que ingresan a las carreras de pedagogía; la calidad de los programas de formación caracterizada por una fuerte separación entre la formación teórica y práctica; la escasa vinculación con establecimientos escolares; y una deficiente formación para trabajar en contextos de alta vulnerabilidad. 
La Organización de las Naciones Unidas para la Educación, la Ciencia y la Cultura, Unesco (2012) plantea tres estrategias para mejorar la formación de profesores:

a. La promoción del ingreso de mejores candidatos a estudiar pedagogía, es decir, elevar el nivel de exigencia de entrada a la profesión.

b. El fortalecimiento de "la calidad de los programas de formación docente, especialmente los contenidos curriculares, estrategias de formación y evaluación de aprendizajes y la calidad de los formados" (p. 119). Para ello plantea elaborar estándares sobre lo que un profesor debe saber y poder hacer.

c. La creación de mecanismos de regulación de la calidad de los programas de formación docente, para lo cual es necesario establecer sistemas de acreditación de las carreras y evaluación de los futuros profesores.

\subsection{Transformaciones en la formación de pregrado}

En Chile, la formación de pregrado se encuentra en debate desde hace más de 10 años, en un proceso que Solar (2005) ha denominado armonización curricular. Las universidades chilenas iniciaron un proceso de transformación mediante la firma de la Declaración de Valparaíso (2003), que adhirió a los principios de la Declaración de Bolonia (2000). De acuerdo con la autora, la firma de la declaración implicó lo siguiente:

a. la promoción de la convergencia del sistema universitario chileno con las transformaciones de los países europeos;

b. el impulso de la adopción de un sistema de créditos compatible entre las universidades chilenas y el sistema de crédito europeo;

c. el fortalecimiento de las relaciones entre universidades y actores externos para mejorar la oferta formativa; $y$

d. la promoción de la movilidad estudiantil y doble titulación (Solar, 2005, p. 173). 
Para enfrentar los desafíos y las demandas a las instituciones de educación superior, el Ministerio de Educación estableció una herramienta de política pública denominada Convenio de Desempeño, que permite financiar iniciativas como la armonización curricular y la formación de profesores. Un Convenio de Desempeño es "un contrato entre el Estado y las Instituciones de Educación Superior (IES) por medio del cual estas comprometen desempeños notables que impliquen un significativo mejoramiento institucional, que por sí solas no habrían podido lograr" (mineduc.cl).

El proceso de renovación curricular se inició en distintas universidades a través de la adjudicación de Convenios de Desempeño, cuyo propósito es la formación de profesores competentes en el logro de aprendizajes de calidad en sus estudiantes. En efecto, los Convenios de Desempeño de Formación Inicial de profesores buscan que las instituciones formen a "los profesores del siglo XXI que Chile necesita, con competencias profesionales de alto nivel que generen cambios notables en la calidad del aprendizaje en las aulas escolares" (mineduc.cl).

\section{Experiencia de rediseño curricular de las carreras de pedagogía}

\subsection{Antecedentes}

La Universidad de Concepción (UdeC) buscó dar respuesta a los nuevos desafíos estableciendo un Plan Estratégico Institucional (2011b) que, en el área de formación de pregrado, se planteó como objetivo consolidar la implementación del modelo educativo UdeC mediante procesos de renovación curricular de todas las carreras. Durante el período 2011-2012, la universidad llevó a cabo tres acciones orientadas a producir un diagnóstico estratégico de las carreras de pedagogía. La primera de ellas consistió en realizar análisis FODA, desarrollado por los Consejos de Carrera, donde participaron profesores y estudiantes.

Una segunda acción se basó en un análisis de los informes de acreditación de las carreras, información que permitió recoger 
las percepciones de distintos agentes involucrados en el proceso de formación de profesores en la UdeC. Un tercer referente estuvo constituido por la revisión de las políticas educativas e informes nacionales e internacionales acerca de la formación inicial docente.

El producto de estos tres análisis arrojó como resultado los siguientes nudos críticos:

- Desactualización de los planes de estudio, duplicidad de contenidos, desalineación con las políticas nacionales y desarticulación entre la formación pedagógica y disciplinar.

- Ausencia de un perfil de egreso alineado con el modelo educativo de la Universidad de Concepción.

- Inexistencia de un sistema formal de prácticas pedagógicas, que gestione el vínculo con los establecimientos escolares, supervise el proceso de inserción de los estudiantes y evalúe su desempeño.

Estos tres nudos críticos justificaron la necesidad de realizar un rediseño curricular en las carreras de pedagogía. A partir de estos resultados y del diagnóstico estratégico institucional, se elaboró un Plan de Acción de Pregrado (2013), cuyo principal objetivo fue asegurar la calidad y la pertinencia de la oferta educativa de la universidad. Para materializar dicho plan, la Dirección de Docencia postuló a fondos del Ministerio de Educación, cuyo resultado fue la adjudicación de un Convenio de Desempeño en el ámbito de formación inicial de profesores, ejecutado entre los años 2013 y 2015. El Convenio de Desempeño "Formación de profesores UdeC: protagonistas del cambio en la sociedad del conocimiento", tuvo como objetivo general "formar profesores de excelencia capaces de demostrar competencias y desempeños notables reflejados en los aprendizajes de calidad y en la formación integral de sus estudiantes" (Universidad de Concepción, 2012). Este convenio contempla nueve objetivos, entre los cuales se encuentra:

Rediseñar el currículo de las carreras de pedagogía para mejorar los aprendizajes de los estudiantes, según un modelo de formación sustentado en el razonamiento y la evidencia científica, el modelo educativo institucional y los referentes 
nacionales e internacionales sobre formación de profesores (p. 23).

Este objetivo fue asumido por comisiones de rediseño de las 19 carreras de pedagogía de la Facultad de Educación del Campus Concepción y de la Escuela de Educación del Campus Los Ángeles 6 .

Es importante mencionar que la Universidad de Concepción definió un modelo educativo donde se incorporan los conceptos de competencias y resultados de aprendizaje, como a continuación se presenta:

Figura 1. Modelo Educativo UdeC

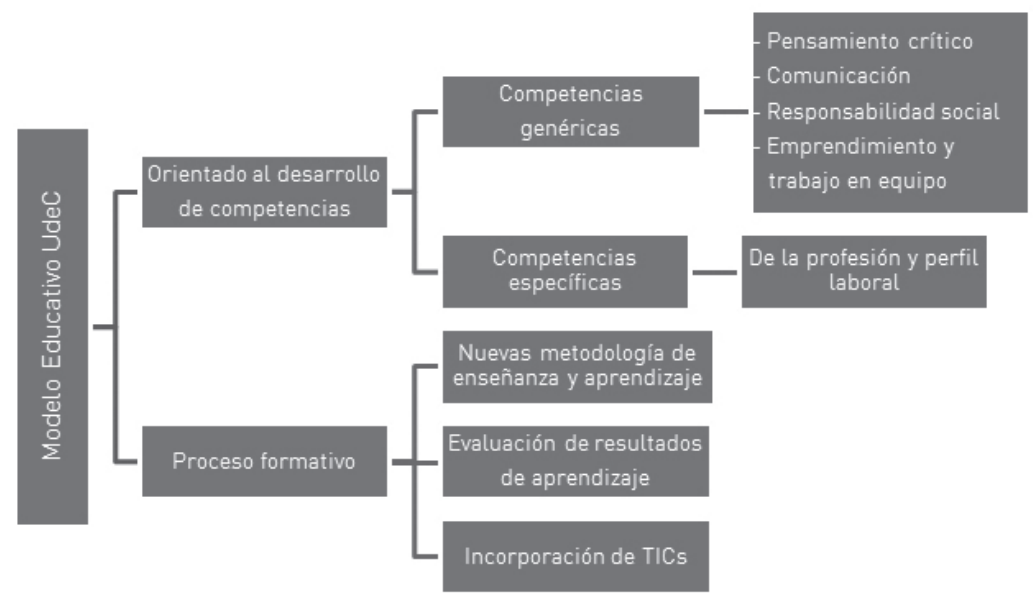

Fuente: Elaboración propia (Universidad de Concepción, 2011a).

\subsection{Etapas del rediseño curricular}

El rediseño curricular se entiende como:

... un proceso que tiene como finalidad que las carreras sean definidas de manera pertinente y adecuada a las necesidades de la sociedad y con altos estándares de calidad. Para lograr este

6 En el Campus Concepción las carreras de pedagogía en: Español, Historia y Geografía, Educación Musical, Filosofía, Inglés, Artes Plásticas, Educación Física, Ciencias Naturales y Física, Ciencias Naturales y Química, y Matemáticas. En el Campus Concepción y Los Ángeles: Pedagogía en Ciencias Naturales y Biología, Educación General Básica, Educación Parvularia y Educación Diferencial. 
propósito, se consideran las características propias de nuestra institución, los lineamientos del nuevo modelo educativo, las características de nuestros estudiantes y las tendencias nacionales e internacionales en educación superior (Unidad de Investigación y Desarrollo Docente, 2013, p. 36).

La Universidad de Concepción definió un método de trabajo para rediseñar las carreras de pregrado que considera tres etapas, como se muestra en la siguiente figura:

Figura 2. Etapas del rediseño curricular

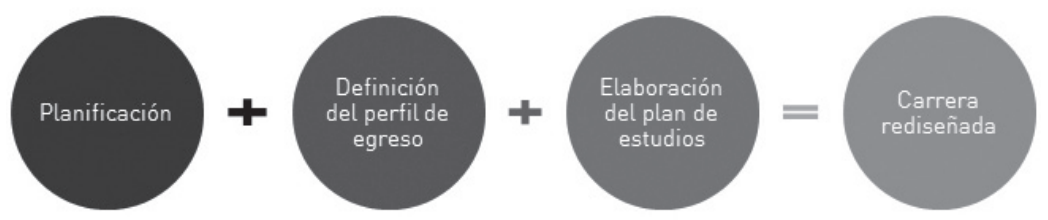

Fuente: Elaboración propia sobre la base de información contenida en UNIDD, 2013.

\section{Etapa 1: Planificación}

En esta etapa el equipo encargado del rediseño curricular de cada carrera formuló un proyecto de rediseño curricular que posteriormente presentó a la Dirección de Docencia para su aprobación, con el fin de iniciar formalmente el proceso. En este proyecto se adoptaron acuerdos, se programaron las fechas para cada etapa del rediseño y se establecieron responsabilidades. Los equipos de trabajo estaban compuestos por el jefe de carrera, académicos de la Facultad de Educación y facultades disciplinares, representantes estudiantiles y un asesor curricular de la Dirección Docencia. El rol del asesor curricular fue acompañar el proceso de rediseño, asegurando el correcto desarrollo de cada etapa.

\section{Etapa 2: Definición del perfil de egreso}

En esta etapa cada equipo de rediseño se encargó de recopilar información de distintas fuentes como referentes teóricos, estándares pedagógicos y disciplinares del Ministerio de Educación, el Marco para la Buena Enseñanza y los planes y programas de estudio de la enseñanza básica y media. En función de esta información y 
del análisis estratégico de cada carrera, se reflexionó acerca de las características deseables que debían presentar los egresados. Para ello se analizó, al interior de cada comisión de rediseño, el estado del arte de los perfiles de otras instituciones nacionales y extranjeras, y se determinó una serie de competencias específicas y genéricas que debía incorporar cada perfil. Se trabajó en una matriz de tributación donde cada competencia era respaldada por un conjunto de resultados de aprendizaje que generaban las asignaturas del nuevo plan de estudios. Esta discusión fue bastante enriquecedora, debido al nivel de interacción que se dio entre los distintos integrantes que participaron, docentes del área disciplinar y académicos de la Facultad de Educación. Este trabajo sistematizado fue un proceso de diálogo permanente, altamente participativo y de revisión continua, donde se generaron instancias de participación de todos los docentes y estudiantes para involucrarlos en cada etapa.

En la redacción tanto de las competencias específicas y genéricas, como de los resultados de aprendizaje fue necesario capacitar a los académicos en el manejo exhaustivo del lenguaje apropiado para explicitar acciones observables y medibles, que dieran cuenta de la conducta esperada de los egresados de cada programa. Este trabajo fue siempre coordinado por el asesor curricular, quien en cada reunión tuvo la función de revisar permanentemente los avances, clarificar conceptos y apoyar en la justificación de cada competencia. Es importante mencionar que las competencias genéricas están establecidas en el Modelo de la Universidad y son cuatro: pensamiento crítico, emprendimiento y trabajo en equipo interdisciplinario, comunicación, y responsabilidad social (Universidad de Concepción, 2013b).

Este proceso culminó con la elaboración de un perfil de egreso preliminar para cada carrera, constituido por un conjunto de competencias (genéricas y específicas), asociadas a dos ámbitos de desempeño: docencia en el sistema educativo, y planificación y desarrollo de proyectos de investigación.

Los equipos de rediseño organizaron, posteriormente, una actividad de socialización con autoridades, académicos y estudiantes 
para presentar el perfil preliminar y recoger observaciones y propuestas para construir el perfil de egreso definitivo. Asimismo, el perfil preliminar se sometió a validación, mediante encuestas en línea aplicadas a académicos, estudiantes, empleadores y egresados. El siguiente gráfico es un ejemplo del proceso de validación.

Gráfico 1. Validación del perfil de egreso de la carrera de Educación Diferencial

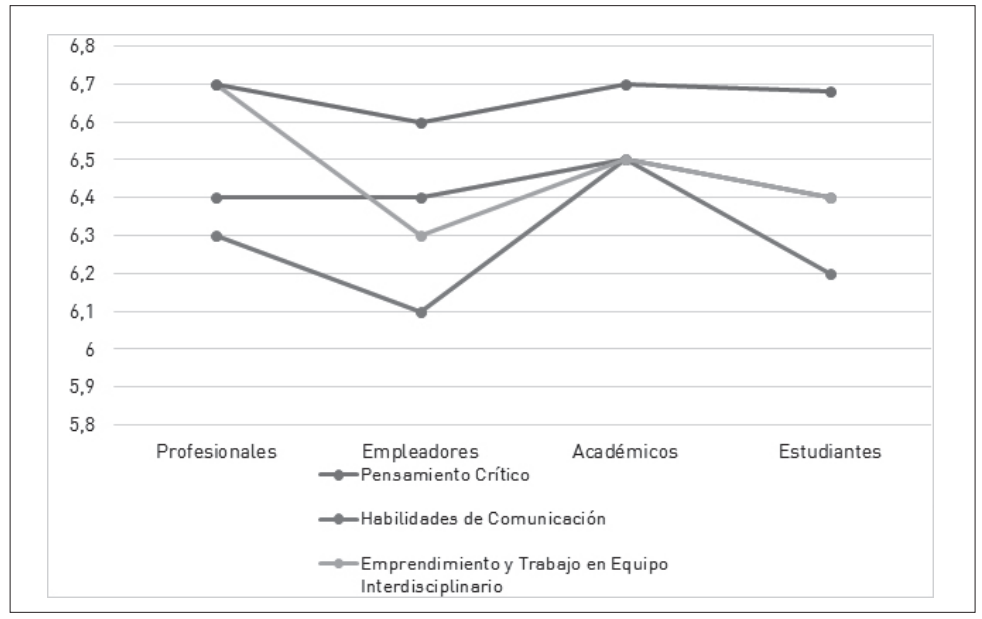

El Gráfico 1 muestra que las competencias genéricas del Modelo Educativo UdeC son ampliamente validadas por los cuatro actores encuestados de la carrera de Educación Diferencial. En efecto, todas las competencias presentan una relevancia sobre 6 , en una escala de 1 a 7 , donde se destaca que la responsabilidad social alcanza la validación más alta, con un promedio de 6,7.

El análisis estadístico de los resultados permitió respaldar la toma de decisiones para mantener, incorporar, modificar o eliminar las competencias, tanto genéricas como específicas del perfil preliminar. Finalmente, el perfil de egreso validado fue dado a conocer públicamente en un Consejo de Carrera ampliado y aprobado por la Facultad de Educación. El cierre de esta etapa se respaldó mediante la firma de un acta oficial que estableció la sanción del perfil de egreso. 


\section{Etapa 3: Definición del plan de estudios}

La Universidad de Concepción define el plan de estudios como un "conjunto de asignaturas y actividades curriculares ordenadas en una secuencia preestablecida conducente a la obtención de un grado académico o un título profesional" (Unidad de Investigación y Desarrollo Docente, 2013, p. 36). Esta etapa consistió en organizar las competencias del perfil de egreso y los resultados de aprendizaje, para luego diseñar los programas de asignaturas y determinar la carga de trabajo académico del estudiante en cada asignatura mediante la asignación de créditos.

El plan de estudio de las carreras de pedagogía se estructuró en torno a cuatro ejes: formación pedagógica, general, disciplinar y práctica. En cada uno de los ejes se diseñaron nuevas asignaturas o se actualizaron los contenidos de las ya existentes, respondiendo a las competencias del perfil de egreso y a los resultados de aprendizaje. Las principales innovaciones se generaron en el eje de formación general y práctica. En el eje de formación general, en primer lugar, se agregó a los planes de estudio la asignatura de Español Académico, destinada a nivelar las competencias de los estudiantes en la habilidad de comunicación. En segundo lugar, la asignatura de Tecnologías de Información se reformuló como una asignatura en modalidad b-learning, considerando lineamientos internacionales acerca del uso de TICs para la enseñanza. 


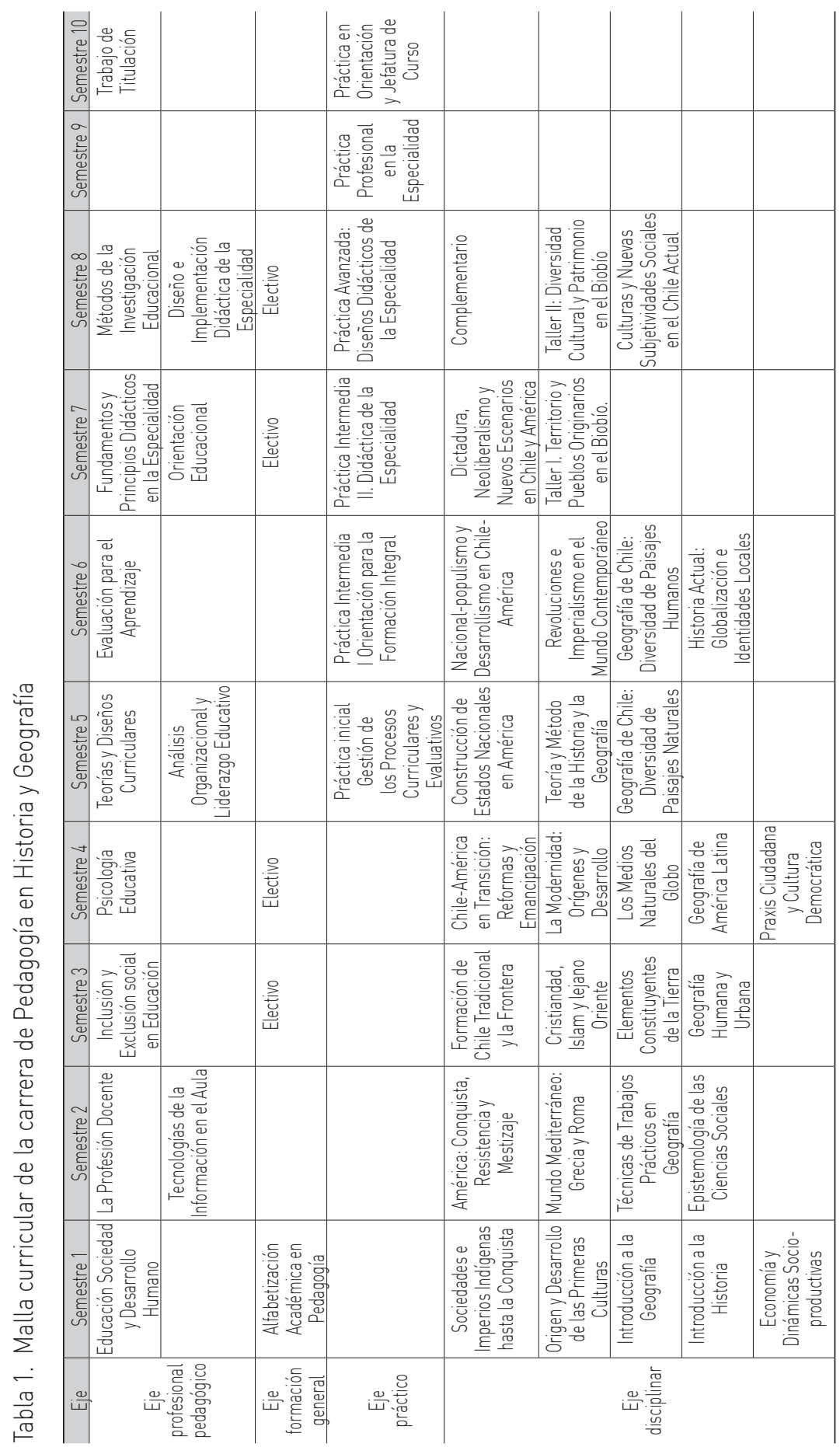


Tabla 2. Distribución de las prácticas pedagógicas

\begin{tabular}{|c|c|c|c|c|c|c|c|c|c|}
\hline Carreras & I Semestre & $\begin{array}{c}\| \\
\text { Semestre }\end{array}$ & $\begin{array}{c}\text { III } \\
\text { Semestre }\end{array}$ & $\begin{array}{c}\text { IV } \\
\text { Semestre }\end{array}$ & V Semestre & $\begin{array}{c}\mathrm{Vl} \\
\text { Semestre }\end{array}$ & $\begin{array}{c}\text { VII } \\
\text { Semestre }\end{array}$ & $\begin{array}{c}\text { VIII } \\
\text { Semestre }\end{array}$ & $\begin{array}{c}\text { IX } \\
\text { Semestre }\end{array}$ \\
\hline $\begin{array}{l}\text { Educación } \\
\text { Parvularia }\end{array}$ & $\begin{array}{l}\text { Práctica } \\
\text { Inicial I }\end{array}$ & $\begin{array}{l}\text { Práctica } \\
\text { Inicial II }\end{array}$ & $\begin{array}{c}\text { Práctica } \\
\text { Intermedia I }\end{array}$ & $\begin{array}{c}\text { Práctica } \\
\text { Intermedia II }\end{array}$ & $\begin{array}{c}\text { Práctica } \\
\text { Intermedia III }\end{array}$ & $\begin{array}{c}\text { Práctica } \\
\text { Avanzadal }\end{array}$ & $\begin{array}{c}\text { Práctica } \\
\text { Avanzada II }\end{array}$ & \begin{tabular}{c|} 
Práctica \\
Avanzada III
\end{tabular} & $\begin{array}{c}\text { Práctica } \\
\text { Profesional }\end{array}$ \\
\hline $\begin{array}{c}\text { Educación } \\
\text { Básica }\end{array}$ & $\begin{array}{l}\text { Práctica } \\
\text { Inicial }\end{array}$ & $\begin{array}{l}\text { Práctica } \\
\text { Inicial }\end{array}$ & $\begin{array}{c}\text { Práctica } \\
\text { Intermedia }\end{array}$ & $\begin{array}{c}\text { Práctica } \\
\text { Avanzada } \\
\end{array}$ & \begin{tabular}{c|} 
Práctica \\
profesional
\end{tabular} & & & & \\
\hline $\begin{array}{l}\text { Educación } \\
\text { Diferencial }\end{array}$ & & & & $\begin{array}{c}\text { Práctica } \\
\text { Inicial }\end{array}$ & $\begin{array}{c}\text { Práctica } \\
\text { Intermedia | }\end{array}$ & $\begin{array}{c}\text { Práctica } \\
\text { Intermedia II } \\
\end{array}$ & $\begin{array}{c}\text { Práctica } \\
\text { Avanzada I }\end{array}$ & $\begin{array}{c}\text { Práctica } \\
\text { Avanzada II }\end{array}$ & $\begin{array}{c}\text { Práctica } \\
\text { Profesional }\end{array}$ \\
\hline $\begin{array}{c}\text { Educación } \\
\text { Media }\end{array}$ & & & & & $\begin{array}{c}\text { Práctica } \\
\text { Inicial }\end{array}$ & $\begin{array}{c}\text { Práctica } \\
\text { Intermedia | } \\
\end{array}$ & $\begin{array}{c}\text { Práctica } \\
\text { Intermedia || }\end{array}$ & $\begin{array}{l}\text { Práctica } \\
\text { Avanzada } \\
\end{array}$ & $\begin{array}{c}\text { Práctica } \\
\text { Profesional }\end{array}$ \\
\hline
\end{tabular}

El eje de formación práctica constituye una diferencia significativa respecto de los planes de estudio anteriores. En el nuevo diseño, las prácticas de inserción temprana en los establecimientos educativos se transforman en nuevas asignaturas, a diferencia de los antiguos planes de estudio, donde formaban parte de las asignaturas de didáctica de la especialidad.

A continuación se detallan los principales componentes utilizados para elaborar el plan de estudios.

a. Resultados de aprendizaje

Cada una de las competencias del perfil de egreso se asocia a un conjunto de resultados de aprendizaje a desarrollar en cada asignatura. Se formulan como enunciados acerca de lo que se espera que el estudiante demuestre una vez terminado el proceso de aprendizaje.

En la siguiente tabla se presenta una matriz de perfiles/ resultados de aprendizaje: 
Tabla 3. Matriz de perfiles resultados de aprendizaje Pedagogía en Historia y Geografía

Competencia 1: Interpretar las dinámicas sociales, económicas y culturales, reconociendo a sus actores, tensiones y transformaciones a partir del uso y aplicación de categorías conceptuales propias de las ciencias sociales

\begin{tabular}{|c|c|c|c|}
\hline $\begin{array}{l}\text { Resultados de } \\
\text { aprendizaje }\end{array}$ & Área disciplinar & $\begin{array}{l}\text { Ámbito de } \\
\text { desempeño }\end{array}$ & Contenidos asociados \\
\hline $\begin{array}{l}\text { Relacionar las } \\
\text { concepciones de la } \\
\text { Geografía y de Historia } \\
\text { estudiadas con los } \\
\text { marcos temporales que } \\
\text { le dan sentido. }\end{array}$ & $\begin{array}{l}\text { Ciencias } \\
\text { Históricas y } \\
\text { Geografía }\end{array}$ & $\begin{array}{l}\text { Docencia en el } \\
\text { sistema educativo }\end{array}$ & $\begin{array}{l}\text { - Claves epistemológicas y } \\
\text { análisis de la teoría y método } \\
\text { de modelos y paradigmas del } \\
\text { siglo XX. } \\
\text { - Métodos de trabajo en la } \\
\text { investigación geográfica. } \\
\text { - Enfoques cualitativos y } \\
\text { cuantitativos. } \\
\text { - Estructura de una } \\
\text { investigación. } \\
\text { - Alcance de la investigación: } \\
\text { exploratoria, descriptiva, } \\
\text { correlacional, explicativa. }\end{array}$ \\
\hline $\begin{array}{l}\text { Analizar las dimensiones } \\
\text { del proceso de } \\
\text { globalización y las } \\
\text { modalidades de } \\
\text { integración y conectividad } \\
\text { a escala regional y } \\
\text { global y sus efectos } \\
\text { en el desarrollo } \\
\text { socioterritorial. }\end{array}$ & $\begin{array}{l}\text { Ciencias } \\
\text { Históricas y } \\
\text { Geografía }\end{array}$ & $\begin{array}{l}\text { Docencia en el } \\
\text { sistema educativo }\end{array}$ & $\begin{array}{l}\text { - Globalización y conformación } \\
\text { de territorios corporativos: lo } \\
\text { global o capitalismo global. }\end{array}$ \\
\hline $\begin{array}{l}\text { Aplicar las categorías } \\
\text { conceptuales propias } \\
\text { de las ciencias sociales } \\
\text { tales como género, } \\
\text { etnia, generación, poder, } \\
\text { relaciones sociales, entre } \\
\text { otras, al análisis de la } \\
\text { organización social. }\end{array}$ & Ciencias Sociales & $\begin{array}{l}\text { Docencia en el } \\
\text { sistema educativo }\end{array}$ & $\begin{array}{l}\text {-El género como categoría de } \\
\text { análisis social. } \\
\text {-Disciplina y construcción } \\
\text { sociohistórica de lo corporal. } \\
\text {-Lo femenino y lo masculino } \\
\text { como construcción } \\
\text { históricocultural: roles, } \\
\text { identidades y estereotipos. } \\
\text {-Sexualidades y diversidades: } \\
\text { enfoques y nuevas } \\
\text { problematizaciones. }\end{array}$ \\
\hline
\end{tabular}

Fuente: Elaboración propia.

\section{b. Perfiles intermedios}

Para definir los perfiles intermedios se agruparon las competencias junto con los respectivos resultados de aprendizaje. La medición de estas competencias se realizó en las asignaturas integradoras. 


\section{c. Asignaturas integradoras}

En los nuevos planes de estudio de las carreras de pedagogía se establecieron asignaturas integradoras, que forman parte de la malla curricular. Ubicadas en el eje de formación práctica, las asignaturas integradoras constituyen un sistema de verificación progresiva de las competencias del perfil de egreso, y conforman la instancia de vinculación entre los contenidos teóricos y la experiencia práctica que desarrollan los estudiantes en los establecimientos educacionales. De acuerdo con el Modelo Educativo UdeC, las asignaturas integradoras representan hitos de evaluación de competencias específicas y genéricas de los perfiles intermedios.

En el Cuadro 1 se aprecia la articulación entre los componentes que constituyen el modelo de asignatura integradora en el plan de estudios en la carrera de Pedagogía en Español:

Cuadro 1. Distribución de las prácticas pedagógicas

\begin{tabular}{l} 
Ámbito de desempeño: docencia en el sistema educativo \\
El profesor egresado de la Universidad de Concepción diseña, planifica y evalúa procesos de \\
enseñanza-aprendizaje en los niveles de su especialidad. Para ello, considera las características \\
de los distintos contextos educativos y la diversidad de sus estudiantes. \\
\hline Competencias: \\
1. Reflexionar y argumentar críticamente sobre las bases teórico/prácticas respecto de la profesión \\
docente, el quehacer educacional y el funcionamiento del sistema educativo. \\
2. Planificar, ejecutary evaluar procesos de enseñanza-aprendizaje en suárea profesional, integrando \\
con una postura innovadora y creativa estrategias y recursos tecnológicos multimodales, según las \\
necesidades de variados contextos educativos. \\
3. Reflexionar de forma continua sobre su práctica en el sistema educativo respetando la \\
multiculturalidad de los contextos y las diferencias individuales de sus alumnos, replanteando \\
progresivamente su desempeño profesional. \\
4. Diseñar, ejecutar y comunicar con rigor científico investigaciones educativas de proyecciones \\
pedagógicas, producto de trabajos en equipo inter y transdisciplinarios.
\end{tabular}

Competencia 2. Planificar, ejecutar y evaluar procesos de enseñanza-aprendizaje en su área profesional, integrando con una postura innovadora y creativa estrategias y recursos tecnológicos multimodales, según las necesidades de variados contextos educativos.

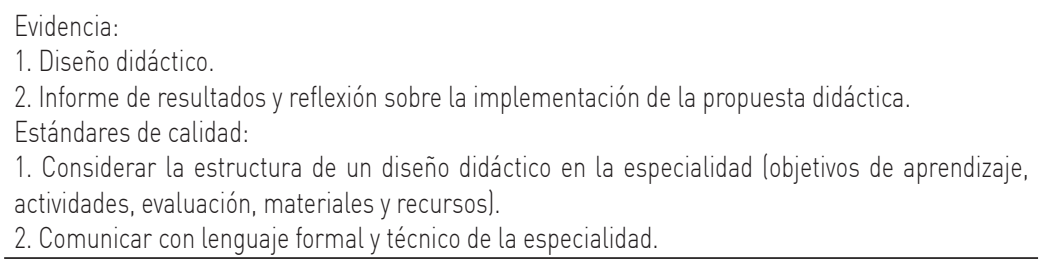

Fuente: Elaboración propia. 


\section{d. Créditos SCT}

Como se mencionó anteriormente, las universidades del Consejo de Rectores definieron un Sistema de Créditos Transferibles (SCT), que propone la asignación de 60 créditos anuales y establece que un estudiante dedique, en promedio, 50 horas semanales a su trabajo académico, considerando horas de trabajo en aula y fuera de ella. Los planes de estudio de las carreras de pedagogía se estructuraron considerando en cada semestre un promedio de 30 créditos SCT, completando un total de 300 créditos SCT en los cinco años de duración. En los programas de asignatura, se distribuyeron los créditos en relación con el trabajo académico del estudiante: horas teóricas, horas de trabajo práctico o laboratorio, y horas de trabajo autónomo.

\section{Conclusiones}

Realizar transformaciones en la formación de profesores implica asumir importantes desafíos en las instituciones de educación superior. Para finalizar este trabajo, se propone analizar la experiencia de rediseño curricular realizado por las carreras de pedagogía de la Universidad de Concepción a través de la propuesta de Zabalza (2008; 2012). De acuerdo con este autor (2008), las innovaciones en estas instituciones conllevan cambios en tres niveles: legal o administrativo, institucional y docencia. En el primer nivel, los cambios se producen mediante nuevas normas. En el caso chileno, la Ley No 20.129 de Aseguramiento de la Calidad de la Educación Superior determina la acreditación obligatoria de las carreras de pedagogía, lo que implica el cumplimiento de ciertos estándares de calidad y la realización de procesos de evaluación y autoevaluación permanentes.

En el segundo nivel, son cambios que se producen en la institución a través de nuevos planes de estudio y dispositivos de evaluación de la docencia. A diferencia del nivel anterior, los cambios se construyen desde la universidad. En el caso de la Universidad de Concepción, la implementación de dos Convenios de Desempeño: "Formación de profesores UdeC: protagonistas del cambio en la sociedad del conocimiento" y "Armonización curricular en la Universidad de Concepción: gestión efectiva del Modelo Educativo Institucional", fue determinante para 
generar transformaciones a nivel institucional en todas las carreras de pregrado. En el caso de las carreras de pedagogía se rediseñó un total de 19 carreras en la Facultad de Educación y en la Escuela de Educación. A este nivel las principales innovaciones realizadas fueron el alineamiento de las todas las carreras con el Modelo Educativo UdeC y la adopción de los créditos SCT.

En el tercer nivel, se encuentra la docencia y la concepción de la enseñanza-aprendizaje. En el proceso de rediseño curricular se buscó incidir en este nivel a través de distintos mecanismos:

a. La realización de talleres de capacitación para apoyar el trabajo de los docentes en el rediseño, tales como redacción de resultados de aprendizaje, diseño de programas de asignaturas, elaboración de syllabus y capacitación en cálculo de SCT.

b. La implementación de cursos para los docentes formadores de profesores en temas relacionados con metodologías de enseñanza, técnicas de evaluación y uso de TICs.

c. El desarrollo de proyectos concursables de apoyo a la docencia destinados a mejorar las prácticas en el aula y favorecer los aprendizajes de los futuros docentes.

d. La realización de pasantías en el extranjero orientadas a profundizar en métodos y estrategias de formación disciplinaria y pedagógica.

Zabalza (2012) plantea, además, que la dificultad central para las innovaciones institucionales y de docencia radica en la transformación de la concepción tanto del currículo como de la enseñanza. "Se trata de un cambio que necesita ser individual, pero que adquiere potencia transformadora solo cuando se convierte en cambio colectivo de manera que se genera una cultura de la innovación" (p. 18).

El proceso de rediseño de 19 carreras de pedagogía implicó grandes desafíos. Por un lado, exigió asumir el contexto de la formación inicial de profesores en Chile, específicamente, la incorporación al currículo de estándares de formación definidos por el Ministerio de Educación y la utilización de créditos transferibles. 
Por otro lado, demandó posesionarse frente a problemas internos, como la desvinculación entre conocimiento disciplinar y pedagógico, y la necesidad de ajustar el currículo al nuevo modelo educativo de la universidad.

Junto con estos desafíos, el proceso de rediseño no estuvo ajeno a las dificultades propias de una transformación importante. Desde el punto de vista de los actores se destacan dos dificultades. La primera fue la resistencia a la implementación del convenio de un grupo de estudiantes de pedagogía; para superar la desconfianza inicial, las autoridades de la Facultad de Educación negociaron con los estudiantes su incorporación en las comisiones de rediseño. De este modo, cada carrera acordó la participación de dos estudiantes en las comisiones, cuyo rol fue facilitar la comunicación entre los profesores y los estudiantes y, además, aportar su perspectiva respecto del antiguo plan de estudios.

La segunda dificultad se relacionó con los académicos. Las transformaciones constituyen instancias desafiantes no solo para las prácticas docentes, sino también para las creencias. La incorporación de un modelo orientado por competencias y la realización de un rediseño curricular fueron cambios significativos, por tanto, fue necesario implementar distintas medidas. Por un lado, se promovió la actualización de las competencias docentes mediante cursos destinados a mejorar las estrategias de enseñanza-aprendizaje y evaluación. Por otro lado, se fomentó la participación de los docentes tanto en las comisiones de rediseño, como en las reuniones de socialización de rediseño. Un mecanismo para garantizar la participación de todos los profesores involucrados en la formación docente fue la Encuesta de Validación de Perfil de Egreso, que permitió conocer las opiniones de todos los académicos (Díaz et al., 2015). En breve, para superar las resistencias de los actores se impulsó su participación en todas las instancias que la universidad podía disponer, de modo que cada uno de ellos tuviera un rol activo en el resultado del rediseño curricular.

Las principales innovaciones realizadas a partir del rediseño curricular, respecto del modelo previo, son las siguientes. 
a. Perfil del egreso de pedagogía

Las carreras de pedagogía de la Facultad de Educación y la Escuela de Educación, hasta el año 2015, tenían un perfil común que consideraba cinco dimensiones enmarcadas en los cuatro dominios del Marco para la Buena Enseñanza: la dimensión personal, la pedagógica, la sociocultural, la organizacional y de gestión, y la disciplinaria.

Actualmente, el perfil de egreso de cada carrera está conformado por un grupo de competencias definidas de acuerdo con ámbitos de desempeño que, en el caso de las carreras de pedagogía, corresponde a docencia e investigación. El perfil de egreso incluye competencias específicas de la carrera y competencias genéricas adaptadas del Modelo Educativo de la universidad. Las competencias se explicitan y contextualizan en resultados de aprendizaje que se enuncian en los programas de asignaturas, y que deben ser logrados por los estudiantes. Además se establecieron perfiles intermedios, ubicados al término del cuarto y octavo semestre del plan de estudios respectivamente, con el fin de evaluar el nivel de progreso de las competencias definidas (Díaz et al., 2015).

\section{b. Plan de estudios}

Los planes de estudio existentes hasta el año 2015 tenían una duración de 10 semestres lectivos con un total de 180 créditos UdeC. En el nuevo rediseño los planes de estudio mantienen los 10 semestres, pero se incorpora el concepto de créditos transferibles (SCT), que busca medir y distribuir el trabajo académico de los estudiantes entre las diversas actividades curriculares que componen su plan de estudios, considerando el tiempo que requieren para lograr los resultados de aprendizaje. Cada plan de estudios tiene un total de 300 créditos SCT distribuidos en cinco años.

\section{c. Estructura curricular}

Los planes de estudio tienen cuatro ejes: formación general, pedagógica, práctica y disciplinar; a diferencia de los planes anteriores en que existían las áreas de especialidad, educación y formación general. Cada una de estas áreas respondía a las cinco dimensiones del perfil de egreso. 
En los antiguos planes, la formación práctica no se visibilizaba como un área específica en las carreras de pedagogía. Producto del trabajo de rediseño y el diagnóstico estratégico se consideró necesario destacar la importancia de las prácticas pedagógicas. Así, en la nueva estructura curricular, la formación práctica presenta el estatus de asignatura propiamente tal, con horas presenciales en la universidad y trabajo práctico en establecimientos educacionales, y conforma un eje de formación práctica. Esta disposición permite fortalecer el vínculo de la universidad con la comunidad escolar. Las asignaturas de este eje se distribuyen en distintos semestres según la carrera (Díaz et al., 2015). Esta estructura curricular permite relevar las competencias del perfil de egreso, orientado a integrar conocimientos teóricos y habilidades prácticas, propias del ejercicio docente.

\section{d. Trabajo de culminación}

El seminario de título fue reemplazado por un trabajo final que adopta dos modalidades: 1) la elaboración e implementación de un diseño didáctico que puede comprender una unidad de aprendizaje, un proyecto de aula, o un módulo instruccional, basado en un contenido relevante en la enseñanza de la disciplina, y 2) un estudio monográfico acerca de un tema, problema, necesidad pedagógica o experiencia educativa. Para el trabajo de culminación es fundamental la vinculación con el sistema educativo, de manera que ambas actividades deben surgir a partir de un problema pedagógico concreto, identificado en algunas de las etapas de las prácticas desarrolladas por el futuro profesor. Esta modalidad busca dar respuesta a una debilidad identificada en el diagnóstico estratégico de las carreras, que reveló que los estudiantes tendían a utilizar más tiempo del establecido oficialmente para finalizar sus carreras, específicamente durante la realización del seminario de título (Díaz et al., 2015).

Una característica importante del rediseño curricular, a diferencia de otras instituciones, es el gran número de carreras de pedagogía involucradas en el proceso: catorce carreras en la Facultad de Educación del Campus Concepción y cinco en la Escuela de Educación del Campus Los Ángeles. Adicionalmente, cada una tiene una vinculación con una facultad disciplinaria que, dada la amplia 
variedad de carreras de pedagogía, incluyen prácticamente a todas estas facultades. Es así como el proceso de rediseño implicó un gran trabajo de coordinación y organización. Un esfuerzo adicional fue realizado por aquellas carreras impartidas en ambos campus: para concretar el rediseño fueron requeridos múltiples viajes y videoconferencias. Los formadores de profesores no solo participaron del rediseño, sino también en las comisiones de cada uno de los programas conformados a partir de los objetivos específicos del convenio de desempeño.

A nivel institucional, cabe destacar que la Universidad de Concepción realizó el rediseño de sus 90 carreras durante la ejecución de dos Convenios de Desempeño: uno destinado a la armonización curricular de todas las carreras, excepto las pedagogías, mencionado anteriormente; y otro destinado exclusivamente a las carreras de pedagogía, una situación que experimentaron pocas universidades. La realización de dos convenios complementarios permitió el desarrollo de líneas de trabajo conjuntas. Entre ellas se destaca la estrategia compartida destinada a "instalar una cultura de análisis y mejoramiento continuo para garantizar la acreditación nacional de carreras" (Universidad de Concepción, 2012, p. 35), que permitió definir nuevos mecanismos para asegurar la calidad de los procesos formativos. La Dirección de Asuntos Estratégicos y la Dirección de Docencia diseñaron un modelo de evaluación de carreras, que busca concretar dicha estrategia mediante la gestión de planes de desarrollo. De acuerdo con este modelo, las carreras deberán revisar sistemáticamente procesos y resultados asociados a la formación. (Díaz et al., 2015). Los análisis derivados de la acreditación institucional y por carreras, unidos al desarrollo de este modelo, buscan generar condiciones que garanticen la calidad de la formación que reciben los estudiantes en la universidad. Asimismo, permitirá consolidar el seguimiento de la implementación del rediseño curricular vigente desde 2016. 


\section{Referencias}

Consejo de Rectores de las Universidades Chilenas, CRUCH. (2013). Manual para la implementación de Sistema de Créditos Académicos Transferibles. SCT-Chile. Santiago de Chile: Consejo de Rectores de las Universidades Chilenas.

Díaz, C., Cisterna, C., Rivas, A., Rojas, C., Soto, V., y Vergara, J. (2015). Innovación curricular en las carreras de pedagogía de la Universidad de Concepción: experiencias y desafíos. En R. Del Valle, P. Molina, y E. Altieri (Eds.), Experiencias de implementación del SCT-Chile (pp. 82-110). Santiago de Chile: Consejo de Rectores de las Universidades Chilenas.

Dochy, F. \& Nickmans, G. (2005) Competentiegericht opleiden en toetsen: theorie en praktijk van flexibel leren. Lemma: Utrecht.

Hambur, S., Rowe, K., \& Lucgrew, L. T. (2002). Graduate skills assessment: Stage one validity study. Canberra: Department of Education, Science and Training.

Ley No 20.129. Establece un Sistema Nacional de Aseguramiento de la Calidad de la Educación Superior del Ministerio de Educación. Publicada en el Diario Oficial del 17 de noviembre de 2006.

Ministerio de Educación, Mineduc (n.d.). En ¿Qué es un Convenio de Desempeño? Financiamiento institucional. Recuperado de http://www.mecesup.mineduc.cl/index2.php?id_portal=59\&id_ seccion $=3605$ \&id_contenido $=14986$

Ministerio de Educación, Mineduc (n.d.). En Ámbito formación inicial de profesores. Financiamiento institucional. Recuperado de http://www. mecesup.cl/index2.php?id_contenido=15048\&id_portal=59\&id_ seccion $=3606$

Navarro, R. (Presidente) (2005). Informe comisión sobre formación inicial docente. Santiago. Recuperado de http://www.oei.es/pdfs/info_ formacion_inicial_docente_chile.pdf

Organization for Economic Co-operation and Development, OECD. (2011). Building aigh-quality teaching profession. Lessons from around the world. New York: Organization for Economic Co-operation and Development.

Organización de las Naciones Unidas para la Educación, la Ciencia y la Cultura, Unesco. (2012). Antecedentes y criterios para la elaboración de políticas docentes en América Latina y el Caribe. Santiago de Chile: OREALC, Unesco. Recuperado de http://unesdoc.unesco.org/ images/0022/002232/223249S.pdf 
Solar, M. (2005). El currículum de competencias en la educación superior: desafíos y problemática. Pensamiento Educativo, 36, 172-191.

Universidad de Concepción (2011a). Modelo educativo Universidad de Concepción. Concepción: Universidad de Concepción.

Universidad de Concepción (2011b). Plan Estratégico Institucional 2011-2015. Concepción: Universidad de Concepción

Universidad de Concepción (2012). Convenio de desempeño UCO 1203: Profesores UDEC: Protagonistas del cambio en la sociedad del conocimiento. Concepción: Universidad de Concepción.

Universidad de Concepción (2013a). Plan de Acción de Pregrado 2011-2015. Documento sin publicar. Concepción: Universidad de Concepción.

Universidad de Concepción (2013b). Modelo de enseñanza de competencias genéricas. Programa de estudios sobre la responsabilidad social. Programa competencias genéricas convenio de desempeño UCO1204. Concepción: Universidad de Concepción.

Universidad de Deusto (2006). Una introducción. Tuning Educational Structures in Europe. La contribución de las universidades al proceso de Bolonia. Bilbao: Publicaciones de la Universidad de Deusto.

Vaillant, D. (2013). Formación inicial del profesor en América Latina: dilemas centrales y perspectivas. Revista Española de Educación Comparada, 22, 185-206. http://dx.doi.org/10.5944/reec.22.2013.9329

Zabalza, M. (2008). Innovación en la enseñanza universitaria: el proceso de convergencia hacia un espacio europeo de educación superior. Educaçao, 31(3), 199-209.

Zabalza, M. (2012). Articulación y rediseño curricular: el eterno desafío institucional. Revista de Docencia Universitaria, 10(3), 17-48.

Recibido: 30/06/2015

Aceptado: 11/05/2016 